\title{
Zur Kenntnis der Nitrochlortoluole
}

\section{Doctoral Thesis}

\section{Author(s):}

Gindraux, Louis

Publication date:

1929

Permanent link:

https://doi.org/10.3929/ethz-a-000092071

Rights / license:

In Copyright - Non-Commercial Use Permitted 


\section{ZUR KENNTNIS \\ DER NITROCHLORTOLUOLE}

Der

Eidgenössischen Technischen Hochschule in Zürich zur Erlangung der

Würde eines Doktors der technischen Wissenschaften vorgelegte

Promotionsarbeit

von

\section{LOUIS GINDRAUX}

Dipl. Ingenieur-Chemiker E. T. H.

Nr. 584

Referent: Herr Prof. Dr. H. E. Fierz

Korreferent: Herr Prof. Dr. Winterstein

B ASEL

Buchdruckerei Emil Birkhäuser \& Co.

1929 
Die vorliegende Arbeit wurde vom Herbst 1927 bis zum Frühling 1929 im Technisch-chemischen Laboratorium der Eidgenössischen Technischen Hochschule ausgeführt.

Es ist mir eine angenehme Pflicht, an dieser Stelle meinem hochverehrten Lehrer

Herrn Prof. Dr. H. E. FIERZ-DAVID,

auf dessen Anregung hin diese Arbeit unternommen wurde, meinen wärmsten Dank auszusprechen für das rege Interesse und die vielseitige Unterstützung, die er mir jederzeit zuteil werden liess.

Sonderabdruck aus Helvetica Chimica Acta, Volumen XII Fasciculus Quintus. 


\section{MEINER LIEBEN MUTTER \\ IN DANKBARKEIT GEWIDMET}




\section{Leer - Vide - Empty}




\section{Zur Kenntnis der Nitro-chlor-toluole.}

Die Derivate des Chlor-toluols haben in den letzten Jahren eine derartige technische Bedeutung erlangt, dass es wünschenswert schien, ihre chemischen und physikalischen Eigenschaften einmal genau zu untersuchen. Die Angaben der Literatur erweisen sich bei genauer Prüfung als unvollständig und, wie ich zeigen konnte, in verschiedenen Fällen als ungenau. Die in Frage kommenden Verbindungen werden hergestellt aus drei verschiedenen Verbindungstypen. Man geht aus entweder von den Toluidinen, oder dann von den Chlor-toluolen, und schliesslich auch von den Nitro-toluolen. In keinem einzigen Falle verläuft die Reaktion einheitlich, und es ist in allen Fällen nötig, die erhaltenen Isomeren durch geeignete Operationen $\mathrm{zu}$ trennen. Das geschieht auf physikalische oder chemische Art; aber ich möchte zum voraus bemerken, dass die Destillation im Vakuum unter Verwendung einer gut wirkenden Dephlegmationskolonne die beste und einfachste Methode darstellt.

Die Verbindungen, welche in Frage kommen, sind folgende:<smiles>C[C]1CCCCC1</smiles><smiles>CC1CCCCC1</smiles><smiles></smiles><smiles>CC1CCC([N+](=O)[O-])CC1</smiles><smiles>CC1CCCCC1[N+](=O)[O-]</smiles><smiles>C[C@H]1CCCCC1=O</smiles><smiles>CC1CCCCC1[N+](=O)[O-]</smiles><smiles>C[C@H]1CCC[C@@H](O)C1</smiles><smiles>C[N+]1(C)C[C]CC1</smiles><smiles>C[N+]1([O-])CCCCC1</smiles> 
Die Herstellung der beiden nitrierten Chlor-toluole erfolgt bekanntlich entweder ausgehend von den entsprechenden Toluidinen, oder dann geht man aus von den Sulfosäuren des Toluols, welche man nach D. R. P. 287932') in die Chlor-toluole überführt. Neuerdings ist auch die elegante Methode von $A$. $W a h l$ hinzugekommen, D. R. P. $376634^{2}$ ), welche darin besteht, dass man Toluol direkt chloriert und darauf das erhaltene Gemisch partiell sulfuriert, wobei das o-Chlor-toluol fast quantitativ in die Sulfosäure übergeführt wird.

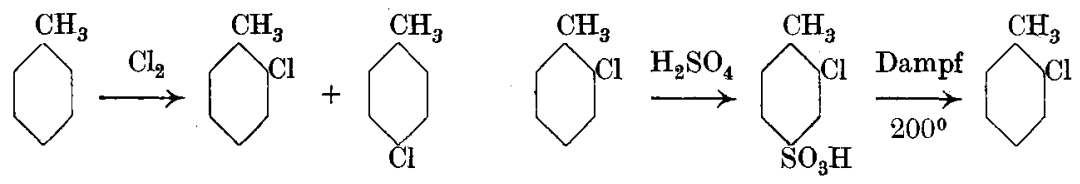

Diese Methode, welche dann später von der J. G. Farbenindustrie $A$. G. auf das Gemisch der beiden isomeren Chlor-nitro-toluole ausgedehnt wurde, ist in dem D. R. P. 434402 ${ }^{3}$ ) eingehend beschrieben und wird durch folgende Formeln versinnlicht:

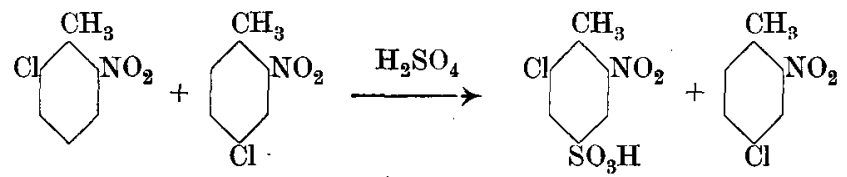

In diesem speziellen Falle handelte es sich weniger darum, eine neue Methode ausfindig zu machen, sondern ich habe versucht, an Hand physikalischer Daten die relativen Mengenverhältnisse der beiden Isomeren in einem gegebenen Gemisch festzustellen. Ich habe gefunden, dass der Brechungsindex hierfür ein befriedigendes Kriterium darstellt, indem z. B. o- und p-Chlor-toluol in ihrer Lichtbrechung so stark verschieden sind, dass eine Analyse auf refraktometrischem Wege bis zu ca. 3\% Genauigkeit möglich ist. Die Trennung ist hier durch Destillation nicht möglich, dagegen wurde gefunden, dass entgegen der Angabe des D. R. P. $434402^{4}$ ) bei den beiden isomeren Chlor-nitro-toluolen 1,2,4 und 1,2,6 eine Trennung durch sorgfältige fraktionierte Destillation ohne grosse Schwierigkeit auszuführen ist. Auch die beiden isomeren Chlor-nitro-toluole 1,2,4 und 1,3,4, welche man durch Nitrierung des $p$-Chlor-toluols erhält, lassen sich verhältnismässig sehr leicht durch fraktionierte Destillation trennen.

Das 2,5-Chlor-toluidin wird ziemlich einheitlich aus dem Acet-otoluidin mit Chlorkalklösung nach der Methode von Claus und Stapelberg ${ }^{5}$ ) erhalten; aber auch hier ist es notwendig, die beiden Isomeren nachträglich durch fraktionierte Destillation oder durch Krystallisation der salzsauren Salze zu trennen.
1) Frdl. 12, 106.
2) Frd1. 14, 377.
3) Frdl. 15, 272.
4) Frdl. 15, 272.
5) A. 274, 287 (1893). 
Der Grund, weshalb ein so grosser Wert auf absolute Reinheit bei der Darstellung gelegt wird, ist darin zu suchen, dass die Technik in den letzten Jahren nur noch mit den chemisch reinen Substanzen arbeitet. Nur dann erhält man bei der Verwendung als Entwicklungskomponente in Kombination mit Naphthol A. S. W. reine Scharlachoder Rot-töne. Auch bei der Überführung der Chlor-toluidine in Thioindigofarbstoffe ist es unbedingt nötig, von vollkommen reinen, einheitlichen Basen auszugehen. Diese zeichnen sich durch charakteristische Schmelz- und Siedepunkte aus, welche zum Teil noch unbekannt waren.

\section{Experimenteller Teil.}

\section{Über die Chlorierung von Benzol, Toluol und ïhren Derivaten.}

Die Chlorierung der aromatischen Kohlenwasserstoffe erfolgt bekanntlich unter Verwendung von trockenem, elementarem Chlor unter Mitwirkung eines geeigneten Katalyten. Es wurde nun gefunden, dass in vielen Fällen, besonders wenn Nitrogruppen vorhanden sind, die Chlorierung langsam und unvollständig verläuft. Auch die Verwendung von Licht verschiedener Art ändert an dieser Tatsache wenig. Es zeigt sich dagegen, dass ein geringer Zusatz von Jod $(0,1 \%$ des Kohlenwasserstoffs) in Kombination mit Eisen (1\%) die Chlorierung ausserordentlich beschleunigt. Im Folgenden sind einige Beispiele aufgeführt und auch graphisch dargestellt. Es ist merkwürdig, dass andere Metalle oder Elemente in Kombination mit Jod sozusagen keine bechleunigende Wirkung ausüben. Es wurden versucht Jod mit Eisen, mit Nickel, mit Kobalt, Antimon, Phosphor, Zinn, Wismut, Zink. Auch die Kombination von drei verschiedenen Katalyten gab kein bemerkenswertes Resultat. Die Verwendung von Eisen und Jod als Katalyt übt nicht nur eine beschleunigende Wirkung aus, sondern die Reaktion verläuft auch bei niedrigerer Temperatur und einheitlich. Aus den erhaltenen reinen Toluolderivaten wurden Zwischenprodukte und Farbstoffe hergestellt. Es seien genannt: Eriochromazurol B (Geigy) und sein Isomeres aus dem 2,4-Dichlor-benzaldehyd, Dichlor-dimethylindigo aus den isomeren Chlor-toluidinen, ferner Dichlor-indigo aus dem 2,6-Nitro-chlor-benzaldehyd, sowie einige Azofarbstoffe vom Typus des Naphthol A. S. der I. G. Farbenindustrie A.-G.

\section{Chlorierung von Benzol.}

Benzol wurde mit Eisen allein als Katalyt chloriert. Die Ausbeute an Monochlorprodukt, bezogen auf wirklich verbrauchtes Benzol, beträgt $80-90 \%$. Die Verluste infolge von Harzbildung steigen bis auf $5 \%$. Der Rest besteht aus Dichlor-benzol.

Die folgende Tabelle gibt Aufschluss über den Reaktionsverlauf. Katalyt, Eisen allein: 
A. Benzol . . . . . 470 g (6 Mol.)

Temperatur. . . . 50-60

Dauer . . . . . . 9 Stunden

Gewichtszunahme . . $125 \mathrm{~g}$

Absolute Gewichtszu-

nahme . . . . $145 \mathrm{~g}$

(20 g Benzol waren während der Chlorierung überdestilliert.)
Destillationsergebnis :

Unverändertes Benzol $180 \mathrm{~g}=2,3 \mathrm{Mol}$.

Monochlor-benzol . . $370 \mathrm{~g}=3,3 \mathrm{Mol}$.

Dichlor-benzol und

$\begin{aligned} \text { Harz . . . . . } & 40 \mathrm{~g}=0,3 \mathrm{Mol} . \\ \text { Verlust . . . . } & =0,1 \text { Mol. }\end{aligned}$

\section{Reaktionsverlauf mit Eisen und Jod als Katalyt:}

B. Benzol . . . . . . $470 \mathrm{~g}$ (6 Mol.)

Temperatur. . . 60-70

Dauer . . . . $31 / 2$ Stunden

Gewichtszunahme . . $140 \mathrm{~g}$

Absolute Gewichtszu-

nahme . . . . $170 \mathrm{~g}$

.C. Benzol . . . . . . $470 \mathrm{~g}$ (6 Mol.)

Temperatur . . unter $20^{\circ}$

Dauer . . . . . 5 Stunden

Gewichtszunahme . . $110 \mathrm{~g}$

Absolute Gewichtszu-

nahme .... $130 \mathrm{~g}(60 \%)$

D. Benzol . . . . . $625 \mathrm{~g}(8 \mathrm{Mol}$ )

Temperatur. . . 20-30

Daud . . . . . 3 Stunden

Gewichtszunahme . - $165 \mathrm{~g}$

Absolute Gewichtszu-

nahme..... $196 \mathrm{~g}(70 \%)$
Destillationsergebnis:

Unverändertes Benzol $175 \mathrm{~g}=2 \mathrm{Mol}$.

Monochlor-benzol . . $310 \mathrm{~g}=2 \mathrm{Mol}$.

Dichlor-benzol und

Harz . . . . $70 \mathrm{~g}=0,5$ Mol.

Die Mengen von $\mathrm{C}_{6} \mathrm{H}_{5} \mathrm{Cl}: \mathrm{C}_{6} \mathrm{H}_{4} \mathrm{Cl}_{2}$ verhalten sïch wie $1: 0,25$.

Unverändertes Benzol $205 \mathrm{~g}=2,5 \mathrm{Mol}$.

Monochlorbenzol- . . $205 \mathrm{~g}=1,8 \mathrm{Mol}$.

Dichlor-benzol und

Harz . . . . $20 \mathrm{~g}=0,14 \mathrm{Mol}$.

Das Verhältnis des Monochlor-benzols zum

Dichlor-benzol beträgt $1: 0,08$.

Destillationsergebnis:

Unverändertes Benzol $205 \mathrm{~g}=2,5 \mathrm{Mol}$.

Monochlor-benzol . . $450 \mathrm{~g}=4 \mathrm{Mol}$.

Dichlor-benzol und

Harz . . . . $35 \mathrm{~g}=0,25 \mathrm{Mol}$.

Das Verhältnis des Monochlor-benzols zum

Dichlor-benzol beträgt $1: 0,06$.

Bei den Versuchen $A-D$ wurde auf eine prozentuale Ausbeuteberechnung verzichtet; es genügte, das Verhältnis von Monochlorbenzol zu Dichlor-benzol zu erfahren. Durch das Ausschütteln, Waschen und Trocknen der Produkte entstehen Verluste, bzw. die prozentuale Zusammensetzung des Gemisches ändert sich.

Bei dem nun folgenden Versuche wurde die Lösung direkt nach dem Chlorieren vom Katalysator abgegossen und destilliert.

E. Benzol . . . . . . . $470 \mathrm{~g}$ (6 Mol.)

Temperatur . . $20-25^{\circ}$

Dauer . . . . . : 4 Stunden

Gewichtszunahme . $130 \mathrm{~g}$

Überdestilliert . . . $15 \mathrm{~g}$

Absolute Gewichtszu-

nahme... . . $145 \mathrm{~g}(70 \%)$
Unverändertes Benzol $210 \mathrm{~g}=2,6 \mathrm{Mol}$. Monochlor-benzol . . $360 \mathrm{~g}=3,2 \mathrm{Mol}$.

Dichlor-benzol und Harz . . . . $25 \mathrm{~g}=0,17 \mathrm{Mol}$.

Verlust . . . . . $=0,03 \mathrm{Mol}$.

Es wurden 3,4 Mol. Benzol verbraucht, die Ausbeute beträgt also: $94 \%$.

Der Zusatz von Jod zum Eisen als Katalyt erleichtert die Bildung höher substituierter Produkte, beschleunigt die Chlorierung und bewirkt eine vollständige Halogenabsorption. 


\section{$-9-$ \\ Chlorierung von Toluol.}

$735 \mathrm{~g}$ (8 Mol.) Toluol wurden mit $280 \mathrm{~g}$ Chlor (100\% der Theorie) und Jod + Eisen als Katalyt bei $50^{\circ}$ chloriert. Zur schnelleren Bestimmung der einzelnen Bestandteile des Reaktionsgemisches wurde jeweils nur ein entsprechender Teil desselben mit Soda und Wasser gewaschen und in einer Claisen-Widmer-Kolonne destilliert. worden:

Aus $31 \mathrm{~g}$ trockenem Produkt sind folgende Fraktionen erhalten

$$
\begin{aligned}
& \text { Toluol 3,5 g. . . . . . . = }=0,04 \text { Mol. }
\end{aligned}
$$

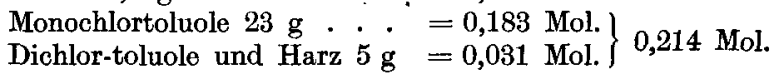

$$
\begin{aligned}
& \text { Dauer: } 4 \text { Stunden. }
\end{aligned}
$$

Die Ausbeute an Monochlor-toluolen beträgt also $85,5 \%$.

Unter gleichen Versuchsbedingungen wurden $650 \mathrm{~g}$ (7 Mol.) Toluol zu $70 \%$ chloriert (170 g Chlor).

Aus $33 \mathrm{~g}$ wurden erhalten:

$$
\begin{aligned}
& \text { Toluol } 7 \mathrm{~g} \text {. . . . . . }=0,08 \mathrm{Mol} \text {. } \\
& \left.\begin{array}{rl}
\text { Monochlor-toluol } 21 \mathrm{~g} \cdot . \dot{ } & =0,166 \mathrm{Mol} . \\
\text { Dichlor-toluol und Harz } 3 \mathrm{~g} & =0,018 \mathrm{Mol} .
\end{array}\right\} 0,184 \mathrm{Mol} \text {. } \\
& \text { Dauer: } 31 / 4 \text { Stunden. }
\end{aligned}
$$

Ausbeute an Chlor-toluol: $90 \%$.

Um einen Anhaltspunkt für die Reaktionsgeschwindigkeit zu haben, wurde der folgende Versuch nur mit Eisen als Katalyt ausgeführt. Unter sonst gleichen Bedingungen ergaben $36 \mathrm{~g}$ Reaktionsprodukt:

$$
\begin{aligned}
& \text { Toluol } 8 \mathrm{~g} \text {. . . . . . }=0,09 \text { Mol. } \\
& \text { Monochlor-toluole 23,5 g . . =0,186 Mol. } \\
& \text { Dichlorprodukte 3,0 g . . . }=0,018 \mathrm{Mol} \text {. } \\
& \text { Dauer der Reaktion: } 7 \text { Stunden. }
\end{aligned}
$$

Die Ausbeute beträgt $91 \%$.

Die Verwendung von Jod + Eisen als Überträger bewirkt hier nicht nur eine Beschleunigung der Chlorierung, sondern sie verhindert auch das Eintreten des Halogens in die Seitenkette, selbst bei Siedehitze. Es ist deshalb hier eine Kühlung nicht notwendig. Die Trennung der beiden Isomeren ist (hier) nur möglich nach D. R. P. 376684 ${ }^{1}$ ) durch Sulfuration des o-Chlor-toluols. Die Sulfogruppe kann nachträglich durch Behandlung mit überhitztem Wasserdampf wieder abgespalten werden.

Die Charakterisierung und Zusammensetzung eines Reaktionsgemisches kann aber viel einfacher aus dem spezifischen Gewicht und aus dem Brechungsindex ermittelt werden.

Handelsprodukte, die zweimal destilliert wurden, ergaben folgende Werte für die Dichte:

1) Frdl. 14, 377. 


\begin{tabular}{|r|c|c|}
\hline & Gefunden & Nach Beilstein \\
\hline \hline $100 \%$ o-Chlor-toluol . . . . & $1,077 / 21^{\circ}$ & $1,081 / 19^{\circ}$ \\
$50 \%$ o-, 50\% p-Chlor-toluol & $1,066 / 21^{\circ}$ & \\
$100 \%$ p-Chlor-toluol . . . . & $1,031 / 20^{\circ}$ & $1,069 / 19^{\circ}$ \\
\hline
\end{tabular}

Trotz vorhergehender Destillation weicht die Dichte des p-Produktes stark vom angegebenen Wert ab, während das aus gleichen Teilen bestehende Gemisch diesem Werte nahekommt. Bei der Chlorierung des Toluols entstehen 60\% o- und 40\% p-Chlor-toluol.

Zur Untersuchung der Brechungsvermögen wurden die Chlortoluole aus den entsprechenden Toluidinen nach Sandmeyer hergestellt; Mischungen davon ergaben folgende Brechungsindices:
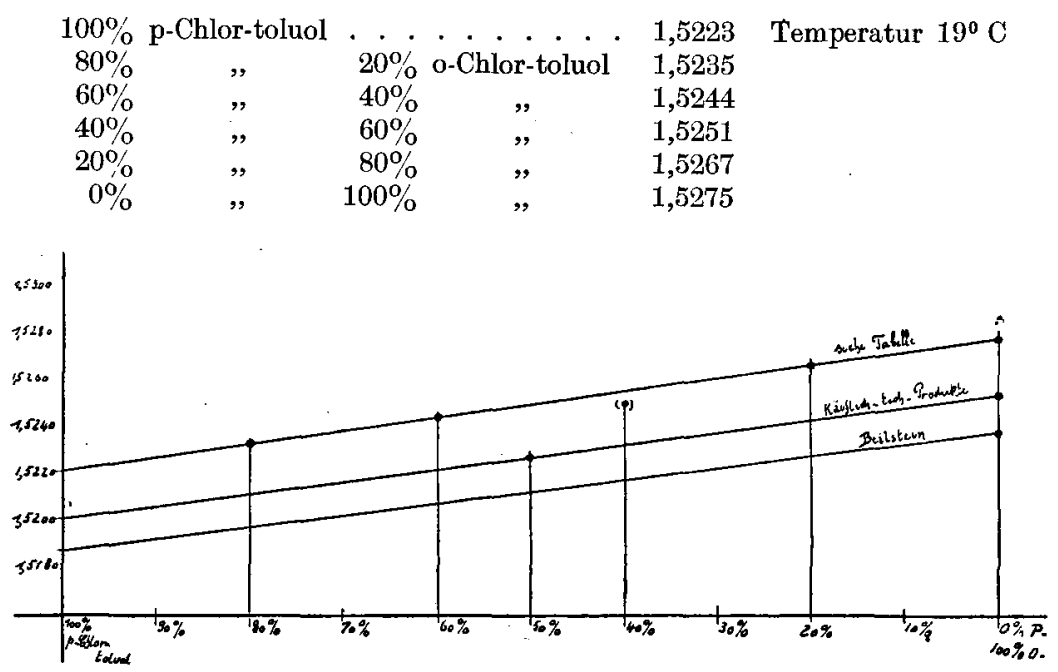

Brechungsindices von verschiedenen Mischungen von o- und p-Chlor-toluol.

Fig. 1.

Alle Werte liegen ungefähr auf einer Geraden, wie aus der graphischen Darstellung ersichtlich ist. Zum Vergleich sind auch die Ergebnisse der käuflichen, technischen Produkte aufgezeichnet, ebenso die Grenzwerte nach den Angaben im Beilstein. Die Proben aus den beiden ersten Chlorierungen ergaben die Werte 1,5248 und 1,5252, und die bei der Destillation der gesamten Reaktionsprodukte bei gleicher 'Temperatur übergegangenen Isomerengemische zeigten die Indices $1,5255-1,5254-$ 1,5254. Sämtliche Werte liegen auf den Kurven der reinen Produkte. Das bei der Hauptdestillation erhaltene Gemisch würde also $60 \%$ ound $40 \% \mathrm{p}$-Chlor-toluol enthalten. Diese Zahlen stimmen mit denen, die in der technischen Literatur angegeben sind, überein. Da bei der Chlo- 
rierung des Toluols kein oder sehr wenig $\mathrm{m}$-Chlor-toluol entsteht und dessen ,Brechungsindex 1,5248 beträgt, so braucht der Einfluss dieses Produktes nicht berücksichtigt zu werden. Durch Bestimmung des Brechungsvermögens hat man die Möglichkeit, die Zusammensetzung des Isomerengemisches schnell zu erkennen.

\section{- Chlorierung von o-Nitro-toluol.}

Zur Chlorierung diente ein Glasgefäss folgender Form:

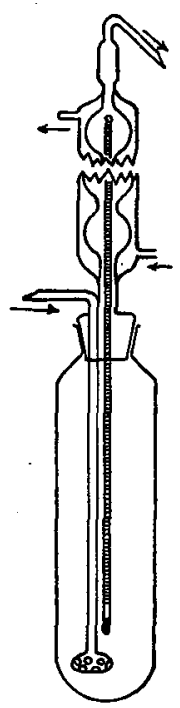

Chlorierungsgefäss

Inhalt 1 Liter.

Fig. 2.

Zur Reaktion kamen 4 Mole o-Nitro-toluol $=548 \mathrm{~g}$. Das Produkt wurde vor der Verwendung destilliert, um alle Feuchtigkeit auszuschliessen. In das Gefäss wurden zuerst $5 \mathrm{~g}$ Eisenpulver und 0,5 g Jod gebracht. Darauf wurde Chlor eingeleitet, um Eisen-Jod-Chlorid zu bilden. Nach einigen Minuten konnte das flüssige Nitro-toluol in den Apparat gegeben und der Einwirkung eines kräftigen trockenen Chlorstromes ausgesetzt werden. Das Eisen-Jod-Chlorid geht nach kurzer Zeit mit dunkelbrauner Farbe in Lösung, und es ist bemerkenswert, dass kein Jod mit den Salzsäuredämpfen entweicht. Die Mischung erhitzt sich sehr rasch, und man kühlt das'Glasgefäss vorteilhaft durch Einstellen in Wasser von $50-60^{\circ} \mathrm{C}$. Es ist auch merkwürdig, dass eine starke Chlorwasserstoffgas-Entwicklung erst dann eintritt; wenn auf $1 \mathrm{Mol}$ Nitro-toluol ungefähr $4 \mathrm{Mol}$. Chlor eingeleitet sind, das Chlor wird vorher nur aufgelöst. Auch das Chlorwasserstoffgas bleibt zum grossen Teil in dem Nitrochlorprodukt gelöst. Aus diesem Grunde ist es nötig, vor der Wägung, behufs Bestimmung des aufgenommenen Chlors, einige Zeit zu evakuieren. Die Chlorierung dauert für die angegebenen Mengen ca. 4 Stunden. Man unterbricht die Chlorzufuhr, wenn $95 \%$ der berechneten Menge aufgenommen sind. Das erhaltene dunkelgefärbte Chlornitroprodukt wird nun mit verdünnter Salzsäure im Scheidetrichter gründlich gewaschen, darauf mit Wasser und nachher mit Lauge von der Säure befreit. Nun destilliert man in der abgebildeten Fraktionierkolonne. Die Trennung erfolgt am besten in Mengen von 2 bis $3 \mathrm{~kg}$. Durch Abkühlung des Dephlegmators sorgt man dafür, dass schätzungsweise auf einen übergehenden Tropfen 15 bis 20 Tropfen in die Kolonne zurückfliessen. Zuerst geht etwas unverändertes Nitro-toluol über, darauf steigt das Thermometer rasch auf $114,6^{\circ} \mathrm{C}$. bei einem Druck von $11 \mathrm{~mm} \mathrm{Hg}$. Der erste Teil des 2,6-Nitro-chlortoluols ist chemisch rein. Ein Teil des 2,4-Nitro-chlor-toluols wird ebenfalls durch Abschleudern des Eutektikums in einer Porzellanzentrifuge chemisch rein erhalten. Um $3 \mathrm{~kg}$ zu destillieren, braucht man ungefähr 24 Stunden. 


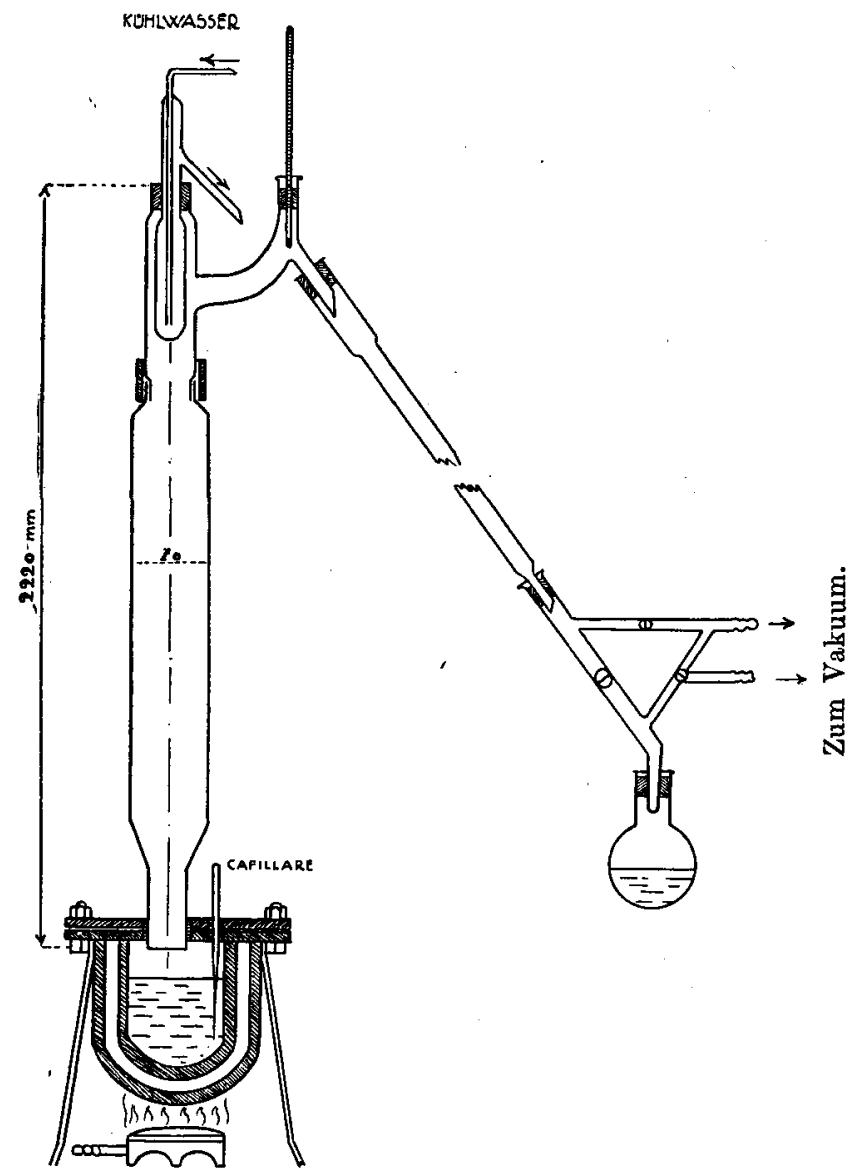

Vakuumdestillationsapparat mit Dephlegmator.

Die Kolonne (aus Mannesmannrohr) enthält 5 Liter Raschigringe $(1 \mathrm{~cm}$ ) aus Glas. Die Säule wird sehr gut isoliert (Wellpappe und Asbest). Der Dephlegmator ist aus Glas und wird mit Asbest und Gummi gedichtet.

Fig. 3.

Folgende Zahlen geben ein Beispiel:

Destilliert:

2350 g Gemisch (aus $14 \mathrm{Mol}$.)

Erhalten: o-Nitro-toluol . . . . . . . $200 \mathrm{~g}$

2,6-Nitro-chlor-toluol (rein) . . $897 \mathrm{~g} \quad$ Smp. $37^{\circ} \quad$ Sdp. 114,6 $11 \mathrm{~mm}$

2,4-Nitro-chlor-toluol . . . . $312 \mathrm{~g}$ Smp. $37^{\circ} \quad$ \# $115,511 \mathrm{~mm}$

Eutektikum . . . . . . . $910 \mathrm{~g}$ Smp. $3^{0}, 5$

Das Eutektikum, welches aus ungefähr gleichen Teilen der beiden Isomeren besteht, konnte unter Verwendung einer Widmer-Kolonne, welche sich für grössere Mengen nicht eignet, weitgehend getrennt wer- 
den. Es entstehen bei der Chlorierung von "o-Nitrotoluol ziemlich genau:

65,9 Teile 2,6-Nitro-chlor-toluol

34,1 Teile 2,4-Nitro-chlor-toluol.

Das Eutektikum wurde jeweils mit der nächsten Chlorierungsoperation vereinigt. Im ganzen wurden ca. $25 \mathrm{~kg} \mathrm{o-Nitro-toluol} \mathrm{chloriert,} \mathrm{und}$ es konnten neben den genannten Produkten keine weiteren beobachtet werden.

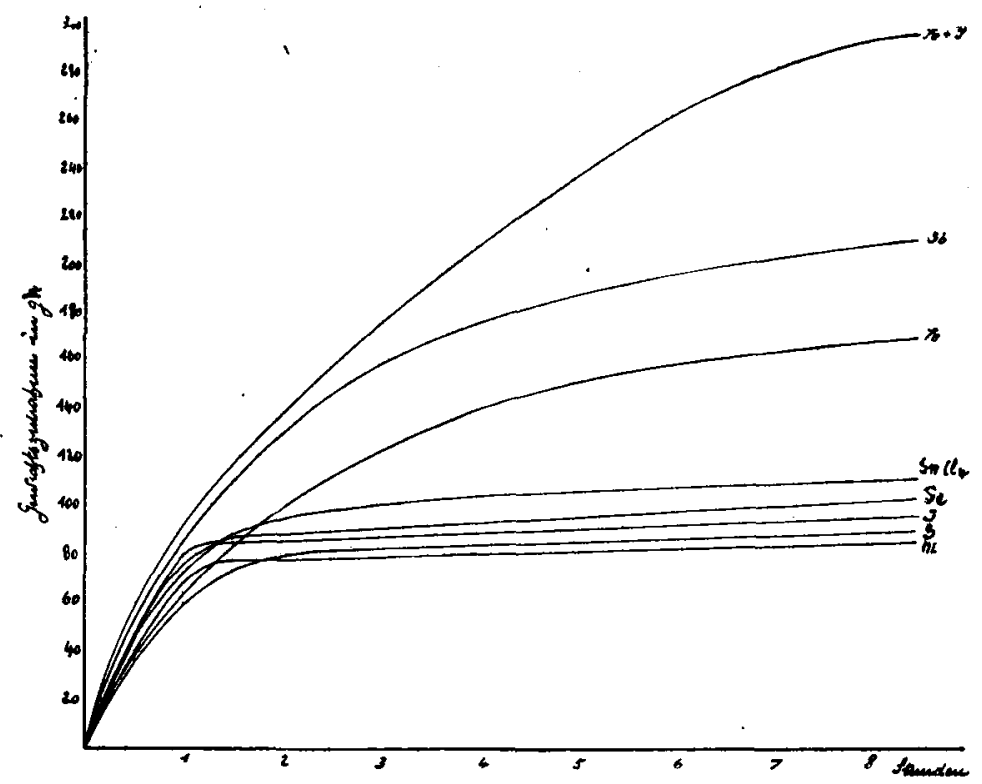

Chlorierung von o-Nitro-toluol, Wirkung verschiedener Katalysatoren. Fig. 4.

Ich möchte noch bemerken, dass die Angaben von Janson, D.R.P. $107505^{1}$ ) ungenau sind, indem er behauptet, dass man bei der Chlorierung von o-Nitro-toluol nur oder fast nur 2,6-Nitro-chlor-toluol erhalte. Die Angaben von Fierz: Grundlegende Operationen der Farbenchemie, S. 100, sind auch unrichtig, indem das Isomere, wie oben erwähnt, das 2,4-Nitro-chlor-toluol ist und nicht das 2,5. Die einzige Angabe, welche den wahren Sachverhalt wenigstens vermuten lässt, findet sich in dem D.R.P. $434402^{2}$ ), wo von 2,4- und 2,6-Nitro-chlor-toluolen gesprochen wird. Dagegen wird dort die unrichtige Behauptung aufgestellt, dass es nicht möglieh sei, die beiden Isomeren durch Destillation zu trennen.

Das 2,6-Nitro-chlor-toluol (Smp. $37^{\circ}$ ) bildet stark doppelbrechende Nadeln mit prismatischer Spaltbarkeit $\left.{ }^{3}\right)$. Bei der optischen Untersuchung der Krystalle ist eine

1) Frdl. 5, 48.

2) Frdl. 15, 272.

3) Die krystallographischen Bestimmungen wurden in freundlicher Weise von Prof. Niggli (Dr. Parker) ausgeführt, wofür wir den Herren auch hier bestens danken. 
gerade Auslöschung feststellbar, die Hauptzone ist negativ. Auf Grund der konoskopischen Untersuchung werden sie als sehr wahrscheinlich optisch einachsig erkannt. (Trigonal, tetragonal, hexagonal.)

Das 2,4-Nitro-chlor-toluol (Smp. $37^{\circ}$ ) bildet nadelige, stark doppelbrechende Krystalle, die äusserlich den 2,6-Nitro-chlor-toluol-Krystallen ähnlich sind. Ausser der prismatischen Spaltbarkeit tritt noch eine basale auf, die im Mittel $15^{0}$ schief zur Pris.menachse steht. Die Auslöschung ist schief, und zwar n/c $20^{\circ} \mathrm{im}$ stumpfen Winkel der basalen Spaltbarkeit. Individuen in spezieller Lage zeigen die basale Spaltbarkeit rechtwinklig zur Prismenachse und löschen gerade aus. Die Krystalle sind also monoklin.

Beide Isomeren lassen sich an Hand der Auslöschungsschiefe ihrer Krystalle ohne weiteres sehr leicht unterscheiden. statt.

Beim Mischen der beiden Isomeren findet sofortige Verflüssigung

Schmelzpunktsdiagramm der eutektischen Mischung von

2,4- und 2,6-Nitro-chlor-toluol.

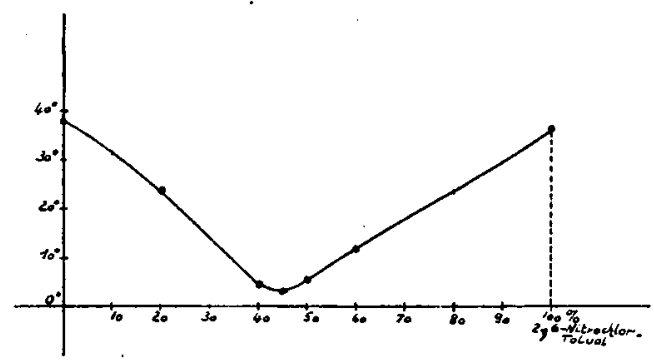

Die maximale Schmelzpunktsdepression liegt nicht beim Verbältnis 1:1. Fig. 5.

\section{Darstellung von 2,6-Nitro-chlor-benzaldehyd.}

Die Chlorierung der Seitenkette des 2,6-Nitro-chlor-toluols verläuft selbst unter Anwendung von Katalysatoren, wie Licht, Phosphorpentachlorid u.a.m. unbefriedigend. Die Versuche wurden ausgeführt bei Temperaturen von $130-210^{\circ}$; aber es war unmöglich, die theoretische Chlorzunahme zu erreichen. Infolge von Wasserbildung wird die Weiterchlorierung erschwert. Es entstehen sehr wahrscheinlich auch Nitrosylverbindungen, oder es können Nitrogruppen abgespalten und teilweise durch Chlor ersetzt werden. Alle diese Erscheinungen verhindern eine glatte Chlorierung der Seitenkette.

$855 \mathrm{~g}$ (5 Mol.) 2,6-Nitro-chlor-toluol wurden unter Bestrahlung 12 Stunden lang bis zu einer Chlorzunahme von $105 \mathrm{~g}$ chloriert. Das gewaschene, dunkelrote, ölige Reaktionsprodukt liess sich durch fraktionierte Destillation nicht befriedigend trennen. Nach D.R.P. 110010 und 115516 wird das Reaktionsgemisch erst nach der Verseifung der Fraktionierung unterworfen. Es lohnt sich aber nicht, nach diesem Verfahren zu arbeiten, weil bei der Chlorierung die Ausbeute an Nitrochlor-benzylchlorid doch zu gering ist. 
Bessere Resultate liefert die Methode von Janson, D.R.P. 107501¹), durch Bromierung der Seitenkette.

$429 \mathrm{~g} \mathrm{(21/2}$ Mol.) 2,6-Nitro-chlor-toluol wurden in Gegenwart von $400 \mathrm{~g}$ o-Dichlor-benzol mit $430 \mathrm{~g}$ Brom bei $165-170^{\circ}$ behandelt. Die nachherige Vakuumdestillation lieferte reines Nitro-chlor-benzylbromid in einer Ausbeute von $87 \%$. (Sdp. $134^{\circ}$ unter $15 \mathrm{~mm} \mathrm{Hg}$; Smp. 50,5 ${ }^{\circ}$.)

Das feste Nitro-chlor-benzylbromid wurde mit 20-proz. Natriumcarbonatlösung (20\% Überschuss) bei $90-95^{\circ}$ glatt verseift. Es empfiehlt sich, diese Operation nicht in Gegenwart des o-Dichlor-benzols auszuführen, weil die spez. Gewichte der beiden Flüssigkeiten zu verschieden sind, um auch bei guter Rührung eine vollständige Aufwirbelung zu erreichen. Der erhaltene Nitro-chlor-benzylalkohol zeigt einen Schmelzpunkt von $56^{\circ}$. Durch Umkrystallisieren aus Ligroin stieg derselbe auf $58^{\circ}$.

Die Oxydation des Alkohols zum Aldehyd wurde mit der theoretischen Menge Bichromat und 20-proz. Schwefelsäure durchgeführt. Durch Reinigung über die Bisulfitverbindung konnten 87\% 2,6-Nitrochlor-benzaldehyd vom Smp. $70,5^{0}$ erhalten werden.

\section{Darstellung von 4,4-'Dichlor-indigo.}

$85 \mathrm{~g}$ Aceton, $42 \mathrm{~g}$ Nitro-chlor-benzaldehyd und $45 \mathrm{~g}$ 1-proz. Natronlauge werden unter Rühren bei $-5^{0}$ gemischt. Nach einer Viertelstunde lässt man $1000 \mathrm{~cm}^{3}$ 1-proz. Lauge bei $20-25^{\circ}$ hinzutropfen, rührt 3 Stunden weiter und lässt über Nacht stehen. Daraufhin können $42 \mathrm{~g}$ Indigoprodukt abfiltriert werden. Durch Umkrystallisieren aus Nitrobenzol werden sehr feine Nadeln erhalten mit gerader Auslöschung und Pleochroismus, blau parallel der Längsrichtung derselben und violett für senkrecht dazu schwingendes Licht ${ }^{2}$ ).

Absorptionsspektrum in Xylol: $\lambda=599,5$.

\section{4,4'-Dichlor-5,5'-dibrom-indigo. Brillantindigo $4 G$.}

Die Bromierung des 4,4'-Dichlor-indigos wurde in Nitrobenzol nach D.R.P. $193438^{3}$ ) ausgeführt.

Absorptionsspektrum in Xylol: $\lambda=614,3$.

Darstellung der Thioindigos aus der TR-Echtscharlach- und KB-Echtrotbase.

Die Darstellung der beiden Farbstoffe geschieht analog derjenigen des Helindonrot $3 \mathrm{~B}$ nach D.R.P. 2419104).

Die diazotierten Chlortoluidine werden mit Alkalixanthogenat gekuppelt, das Kupplungsprodukt verseift und mit Chloressigsäure kondensiert. Die erhaltenen Chlor-methyl-phenyl-thioglykolsäuren kry-

1) Frdl. 5, 50.

2) Die krystallographischen Bestimmungen wurden in zuvorkommender Weise vom Mineralogischen Institut der E. T. H. (Prof. Dr. P. Niggli) ausgeführt.

3) Frdl. 9, 523.

4) Frdl. 10, 502. 
stallisiert man aus Alkohol um und kondensiert sie mit Chlorsulfonsäure zum Farbstoff.

Aus 6-Chlor-o-toluidin wurde in einer Ausbeute von $76 \%$ die 2-Methyl-3-chlor-phenyl-thioglykolsäure vom Smp. $104^{0}$ erhalten.

Der 6, $6^{\prime}$-Dichlor-7, $7^{\prime}$ dimethyl-thioindigo stellt ein rotes Pulver dar, welches in konz. Schwefelsäure mit grüner Farbe löslich ist. Aus alkalischer Hydrosulfitlösung färbt er Baumwolle sehr leicht in hellroten Tönen, Wolle etwas weniger leicht in braunroter Nuance an. Die Färbungen sind sehr gut wasch- und chlorecht und gut lichtecht. Die Ausbeute an Farbstoff betrug 83,5\%. Absorptionsspektrum in Xylol $\lambda=544,8$.

Die 2-Methyl-5-chlor-phenyl-thioglykolsäure (Smp. 1010) wurde mit einer Ausbeute von $84 \%$ erhalten. Der daraus hergestellte 4, $4^{\prime}$ Dichlor-7, $7^{\prime}$-dimethyl-thioindigo ist ein braunrotes Pulver, welches sich gleich wie sein Isomeres verhält. Die Ausfärbungen sind etwas braunstichiger.

Absorptionsspektrum in Xylol $\lambda=553,6$.

Nitrierung von $p$-Chlor-toluol.

Wie aus folgenden Versuchsschemata zu ersehen ist, lässt sich die Bildung von 2,4-Nitro-chlor-toluol auch bei verschiedenen Bedingungen nicht weitgehend steigern.

1. $750 \mathrm{~g} \mathrm{p}$-Chlor-toluol

$1200 \mathrm{~g}$ Schwefelsäure 96-proz.

$375 \mathrm{~g}$ Salpetersäure $(\mathrm{D}=1,52)$

Temperatur: $0-8^{0}$

Dauer: $1 \frac{1}{2}$ Stunden.

Destillationsergebnis :

$145 \mathrm{~g}$ unverändertes p-Chlor-toluol $58,7 \%$ 2,4-Nitro-chlor-toluol $41,3 \%$ 3,4-Nitro-chlor-toluol

Sdp. von 2,4-Nitro-chlor-toluol unter $11 \mathrm{~mm}=115,5^{\circ} \mathrm{C}$

Sdp. von 3,4-Nitro-chlor-toluol unter $11 \mathrm{~mm}=118^{\circ}$

2. $500 \mathrm{~g}$ p-Chlor-toluol

$800 \mathrm{~g}$ Schwefelsäure 96-proz.

$250 \mathrm{~g}$ Salpetersäure $(\mathrm{D}=1,52)$

Temperatur: $8-13^{\circ}$

Destillationsergebnis:

$61 \% 2,4$ - und $39 \%$ 3,4-Nitro-chlor-toluol.

3. $265 \mathrm{~g}$ p-Chlor-toluol

$235 \mathrm{~g}$ Schwefelsäure $66^{\circ}$ Bé

$155 \mathrm{~g}$ Salpetersäure 80-proz.

Temperatur: $1 \frac{1}{2}$ Stunden bei $25-35^{0}$, dann 2 Stunden bei $50^{\circ}$

Destillationsergebnis:

$15 \mathrm{~g}$ unverändertes p-Chlor-toluol

$62,5 \% 2,4$-Nitro-chlor-toluol

$37,5 \%$ 3,4-Nitro-chlor-toluol.

4. $632,5 \mathrm{~g}$ p-Chlor-toluol

700 g Schwefelsäure, konz. Temperatur: 50-60

384 g Salpetersäure, 79-proz.

Destillationsergebnis:

$8 \mathrm{~g}$ unverändertes p-Chlor-toluol

$53 \% 2,4$-Nitro-chlor-toluol

47\% 3,4-Nitro-chlor-toluol. 
Darstellung von m-Nitro-p-toluidin.

Im 3,4-Nitro-chlor-toluol ist das Chloratom infolge seiner Stellung befähigt, sich mit Ammoniak zum entsprechenden Nitro-toluidin umzusetzen.

$57 \mathrm{~g}$ m-Nitro-chlor-toluol wurden im Autoklaven mit $200 \mathrm{~g}$ 30-proz. Ammoniak bei $175^{\circ}$ behandelt. Nach 12 Stunden konnten $45 \mathrm{~g}$ der Base vom Smp. $95^{\circ}$ erhalten werden. Durch Umkrystallisieren des Chlorhydrates aus Wasser stieg der Smp. der Base auf 1140. Ausbeute $=82 \%$.

\section{Darstellung von m-Nitro-p-kresol.}

3,4-Nitro-chlor-toluol lässt sich mit alkoholischen oder wässerigen Alkalien, selbst unter Anwendung von Druck, nicht in das entsprechende Nitro-p-kresol überführen.

Das gesuchte Produkt konnte aber erhalten werden durch $\mathrm{Be}$ handeln von m-Nitro-p-toluidin mit 10-proz. wässeriger Natronlauge. Nach 12-stündiger Reaktionsdauer bei $130^{\circ}$ konnte eine Ausbeute von $90 \%$ an m-Nitro-p-kresol erreicht werden.

\section{Darstellung von Kresidin.}

51 .g $(3 / 10$ Mol. $) \quad$ Nitro-chlor-toluol wurden mit $16,8 \mathrm{~g}$ Kaliumhydroxyd und $200 \mathrm{~cm}^{3}$ Methylalkohol 24 Stunden auf $110^{\circ}$ erhitzt. Der Boden des Autoklaven bedeckt sich mit Kaliumchloridkrystallen, denen etwas Azoxy-kresol (0,5 g, Smp. 1470) beigemengt ist. Nach dem Abdunsten des Methylalkohols wurde die ölige Flüssigkeit mit Wasser gewaschen und im Vakuum destilliert. Bei $122^{\circ}$ unter $12 \mathrm{~mm} \mathrm{Hg}$ gingen $12 \mathrm{~g}$ unverändertes Nitro-chlor-toluol über und bei $144^{\circ}$ unter $12 \mathrm{~mm} \mathrm{Hg}$ destillierten $31 \mathrm{~g}$ Nitro-kresol-methyläther über. Das letzte Produkt wurde in einer Menge von $5 \mathrm{~g}$ in $60 \mathrm{~cm}^{3}$ Alkohol gelöst, mit $60 \mathrm{~cm}^{3}$ konz. Ammoniak versetzt und während 3 Stunden mit einem kräftigen Schwefelwasserstoffstrom reduziert. Die. Lösung färbte sich dabei dunkel und wurde nach einem Zusatz von Soda nach 12-stündigem Stehen ausgeäthert. Durch Behandeln mit wenig Alkohol ging das Kresidin in Lösung und konnte nach der Vakuumdestillation in einer Ausbeute von $42 \%$, als weiss-graues Pulver vom Smp. 50\%, erhalten werden.

\section{Darstellung der Eriochromazurole.}

Die folgenden Produkte wurden nach den Angaben von Fierz: Grundlegende Operationen der Farbenchemie; S. 100, aus den 2,6und 2,4-Nitro-chlor-toluolen hergestellt.

$$
\begin{aligned}
\text { Ausbeute an: } & \text { 2,6-Dichlor-toluol . . . . . } 81 \% \\
& \text { 2,6-Dichlor-benzalchlorid. . } 89 \% \\
& \text { 2,6-Dichlor-benzaldehyd . . } 68 \% \\
& \text { 2,4-Dichlor-toluol . . . . . } 78,5 \% \\
& \text { 2,4-Dichlor-benzalchlorid. . } 91 \% \\
& \text { 2,4-Dichlor-benzaldehyd . . } 69,5 \%
\end{aligned}
$$


Die Kondensation der Aldehyde mit o-Kresotinsäure erfolgte nach D.R.P. $234027^{1}$ ). Die Leukoverbindung wurde mit Nitrosylschwefelsäure zum Farbstoff oxydiert.

Die nachchromierten Färbungen zeichneten sich durch ausserordentliche Reinheit in der Nuance und durch gute Waschechtheit aus.

Farbstoff aus 2,6-Dichlor-benzaldehyd:

Absorptionsspektrum in Natronlauge $\lambda=597,8$

, $\quad$, Schwefelsäure $\lambda=536,3$

"

"Schwefelsäure + Borsäure $\lambda=535,8$ undeutlich.

Farbstoff aus 2,4-Dichlor-benzaldehyd:

Absorptionsspektrum in Natronlauge $\lambda=593,0$

"Schwefelsäure $\lambda=521,5$

Der Farbstoff aus 2,4-Dichlor-benzaldehyd ist, wie schon das Patent von Geigy sagt, sehr farbschwach. Die Färbung ist trüber und viel grüner als jene des Eriochromazurols von Geigy.

1) Vgl. auch D. R. P. 189938, Frdl. 9, 200; 198909, Frdl. 9, 201; 199943, Frdl. 9, 204. 
Ich, Louis Gindraux, wurde am 4. Februar 1905 in Payerne (Kt. Waadt) geboren. In Biel dùrchlief ich die Primar- und Sekundarschule und erwarb mir am Gymnasium das Maturitätszeugnis im Oktober 1923. Anschliessend daran begann ich meine Studien an der Chemischen Abteilung der Eidgenössischen Technischen Hochschule und erhielt im Juni 1927 das Diplom als Ingenieur-Chemiker. Vom Herbst 1927 bis Frühling 1929 beschäftigte ich mich mit vorliegender Promotionsarbeit, die ich im Laboratorium von Herrn Prof. Dr. H. E. Fierz-David ausführte. In der Zeit vom April 1928 bis März 1929 war ich als Assistent am Technisch-chemischen Laboratorium tätig. 